\title{
Is there a relationship between response to total sleep deprivation and efficacy of clomipramine treatment in depressed patients?
}

\author{
D. Höchli, D. Riemann, J. Zulley and M. Berger
}

From the Max-Planck-Institute of Psychiatry (Head: Prof. Dr. D. Ploog), Munich, F.R.G.

\begin{abstract}
Total sleep deprivation (TSD) and tricyclic medication are successful treatment modalities for patients with a major depressive disorder. Recent studies have suggested a positive relationship between TSD response and succeeding tricyclic treatment, even on a very specific level, thus supporting the assumption of two distinct biochemical subtypes of depression. The present study tested this hypothesis by treating 10 inpatients with a major depressive disorder first with TSD and succeedingly with clomipramine. Contrary to expectation, a negative relationship between clinical response to the two treatment modalities was found.
\end{abstract}

Received February 15, 1986; accepted for publication March 1, 1986

Total sleep deprivation (TSD) has become a commonly used tool in the treatment of depressive disorders. In a review article on the literature concerning this issue, Gillin (1) summarized that TSD is a successful treatment intervention in depressed patients, leading to an alleviation of depression in at least $50 \%$ of the patients studied. Nevertheless, therapeutic effects of TSD are mainly confined to the first day following such treatment; in most cases, clinical relapse takes place the next day. Wirz-Justice et al. (2) reported a significant correlation between positive response to TSD on the first day and efficacy of clomipramine treatment, whereas patients responding on the second day to TSD showed good clinical improvement to succeeding treatment with maprotiline. Keeping in mind the pharmacology of these drugs, i.e. that clomipramine inhibits serotonin reuptake, and maprotiline acts via norepinephrine reuptake inhibition, Wirz-Justice et al. (2) speculated that the kind of response to TSD might provide a helpful tool to improve differential-therapeutic treatment with antidepressants. Fähndrich (3) replicated this result, whereas Wirz-Justice et al. (4) and Amin (5) could not confirm this finding. However, a positive correlation between TSD response and response to antidepressant drug treatment in general, regardless of mode of action of drugs under investigation, was confirmed. The aim of the present study is to further clarify the relationship between TSD response and efficacy of succeeding clomipramine treatment.

\section{Methods}

Ten inpatients ( 7 females and 3 males) with a major depressive disorder, endogenous subtype (RDC (6)), three bipolar and seven unipolar, age ranging from 18-59 years (mean: 40.2 years). with a 21-item Hamilton score ranging from 21-36 points (mean \pm SD: $28.2 \pm 5.2$ ) were included in the study. Psychotic patients and patients with a severe suicidal risk were excluded Patients were drug-free for at least 3 weeks prio to TSD and clomipramine treatment. Afrer to TSD and clomipramine treating which a serie
baseline phase of 10 days, during 
afory tests and sleep recordings were laboratory lests underwent a night of TSD. erformed, pafter TSD, patients were rated by an Before and after psychiatrist on the 6-item version of experienced Hamilton scale. Additionally, patients selfrated their mood each morning and evening before and after TSD with a mood adjective checklist, the Befindlichkeits-Skala (Bf-S) (7). Two days after TSD, clomipramine was administered in an oral dose of $75 \mathrm{mg}$ on the first day, then continued throughout the following 18 days with a total dose of $150 \mathrm{mg}$ daily, given in three portions at 8.00 a.m., 12.00 a.m. and 4.00 p.m. To evaluate clinical response to clomipramine rreatment, patients were rated on the 21 -item Hamilton scale during baseline and on the 19th day of treatment. Furthermore, patients selfrated their mood each morning and evening during these days using the Bf-S.

\section{Results}

Establishing a response criterion of at least $30 \%$ improvement or more on the 6-item version of the Hamilton scale, five of 10 patients responded well to TSD. Two patients just slightly improved, whereas the remaining three patients showed a deterioration of mood. Comparing mean Hamilton scores before and after TSD, values decreased from $12.1 \pm 3.0$ to $8.7 \pm 6.1(P<0.07$, Wilcoxon test, one-tailed). A similar effect could be found for self-rating of mood. Mean Bf-S (averaged morning and evening scores) decreased from 42.0 \pm 7.8 to $31.6 \pm 12.9(P<0.05$, Wilcoxon test, one-tailed). The correlation between objective and subjective improvement almost reached statistical significance $(r=0.55, P<0.07$, Spearman rank correlation, one-tailed).

Clomipramine treatment led to significant improvement on the 21-item Hamilton scale (from $28.2 \pm 5.2$ to $16.3 \pm 9.5, P<0.01$, Wilcoxon test, one-tailed). Regarding a response criterion of at least $30 \%$ or more improvement on the Hamilton scale from baseline to the 19th day of medication, six of the patients were considered good responders. Concerning the Bf-S scores, self-rated mood improved from $39.9 \pm$ 9.4 to $30.3 \pm 13.4$ ( $P<0.05$, Wilcoxon test, onetailed).
The correlation between TSD response and clomipramine response, however, was not very high: Only two of five TSD responders responded well to clomipramine. Of the remaining three TSD responders, two just slightly improved, and one worsened during drug treatment. The three patients showing a negative response to TSD improved well during drug treatment. Of the two patients reacting only slightly to TSD, one improved well and the other just slightly to clomipramine. Correlating percentage of improvement during TSD and drug treatment, a significant negative coefficient emerged $(r=-0.66, P<0.05$, Spearman rank correlation, one-tailed), indicating that the better the response to TSD, the lower the therapeutic efficacy of clomipramine.

\section{Discussion}

The presented results concerning the therapeutic effects of either TSD or clomipramine treatment in depressive patients are in good agreement with the literature. TSD led to a clear-cut improvement in $50 \%$ of the patients, whereas clomipramine treatment alleviated depression in $60 \%$ of all the patients studied. Contrary to expectation, no positive relationship between the two treatment modalities could be found; however, a significant negative correlation existed. This clearly contradicts both previously mentioned studies ( 2 , 3). Unfortunately, no objective clinical rating was administered on the 2 nd day following TSD. Regarding subjective mood-rating on that day, it was, however, definite that none of the 1st day non-responders was a 2 nd day responder.

So far, findings of the presented study do not support the assumption put forth by Wirz-Justice et al. (2) and Fähndrich (3) that TSD response in depressed patients might help to identify specific subtypes of depression, one being characterized by a central nervous deficiency in serotonin. According to this theory, all the patients responding well to TSD should have been good clomipramine responders, which was not the case. A recent study by our group (8) tested, in a double-blind cross-over design, the therapeutic efficacy of oxaprotiline, a specific norepinephrine reuptake inhibitor, vs. fluvoxamine, a 
specific serotonin reuptake-inhibiting compound, in patients with a major depressive disorder. Results of this study clearly contradict the hypothesis of two distinct biochemical subtypes of depression. It was found that non-responders to one of the drugs mostly were also nonresponders to the alternative antidepressant. The more general assumption of Wirz-Justice et al. (4) that positive TSD response constitutes a predictor for succeeding improvement under tricyclic medication, regardless of the mode of action of these drugs, i.e. whether specific serotonin vs. norepinephrine reuptake inhibition, could also not be confirmed.

However, considering the small sample size in the present study, further replication and validation is suggested in order to draw decisive clinical and theoretical conclusions.

\section{References}

1. Gillin J C. The sleep therapies of depression. Progr Neuropsychopharmacol Biol Psychiatry 1983:7:351-364.

2. Wirz-Justice A, Pühringer W, Hole G. Sleep deprivation and clomipramine treatment in endogenous depression. Lancet 1976:2:350.
3. Fähndrich E. Effect of sleep deprivation as a predictor of treatment response to antidepressant medication. Acta Psychiatr Scand 1983:68:341-344.

4. Wirz-Justice A, Pühringer W, Hole G. Response to sleep deprivation as a predictor of therapeutic results with antidepressant drugs. Am J Psychiatry 1979:136:1222-1223.

5. Amin M. Response to sleep deprivation and therapeutic results with antidepressants. Lancet 1978:2:165.

6. Spitzer R L, Endicott J E, Robins E. Research diagnostic criteria for a selected group of functional disorders, $3 \mathrm{rd}$ ed. New York: National Psychiatric Institute, Biometric Research, 1977.

7. Zerssen D von, with the assistance of Koeller D M. Die Befindlichkeits-Skala. Weinheim: Beltz, 1976.

8. Emrich H, Berger M, Riemann D, von Zerssen D. Serotonin vs. norepinephrine inhibition: A double-blind differential therapeutic study with fluvoxamine and oxaprotiline in endogenous and neurotic depressives. Pharmacopsychiatry 1986 (submitted for publication).

\section{Address}

Mathias Berger, M.D.

Max-Planck-Institute of Psychiatry

Kraepelinstr. 10

D-8000 München 40

F.R.G. 